\title{
Comparison of B2-EIRENE calculations with multi-machine experimental measurements
}

\author{
A. Loarte ${ }^{\text {a,* }}$, A.S. Kukushkin ${ }^{\text {b }}$, H.D. Pacher ${ }^{\text {a }}$, D.P. Coster ${ }^{\text {c }}$, R. Schneider ${ }^{\text {c }}$, \\ N. Asakura ${ }^{\text {d }}$, K. Itami ${ }^{\text {d }}$, B. LaBombard ${ }^{\text {e }}$, B. Lipschultz ${ }^{\text {e }}$, C.F. Maggi ${ }^{f}$, \\ R.D. Monk ${ }^{\mathrm{f}}$, G.D. Porter ${ }^{\mathrm{g}}$, M. Shimada ${ }^{\mathrm{d}}$, M. Sugihara ${ }^{\mathrm{b}}$, J. Terry ${ }^{\mathrm{e}}$ \\ ${ }^{a}$ The NET Team, Max-Planck-Inst. für Plasmaphysik, Boltzmanstrasse 2, D-85748, Garching, Germany \\ b ITER Joint Central Team, Joint Working Site, D-85748, Garching, Germany \\ ${ }^{c}$ Max-Planck-Institut für Plasmaphysik, EURATOM Association, D-85748, Garching, Germany \\ d JAERI, Naka Fusion Research Establishment, Ibaraki-ken 311-01, Japan \\ e Plasma Fusion Centre, MIT, Cambridge, MA 01239, USA \\ ${ }^{\mathrm{f}}$ JET Joint Undertaking, Abingdon, OX14 3EA, UK \\ ${ }^{g}$ Lawrence Livermore National Laboratory, P.O. Box 808, Livermore, CA 94550, USA
}

\begin{abstract}
The B2-EIRENE code has been compared with plasma edge measurements from Alcator C-MOD, DIII-D, JET, JT$60 \mathrm{U}$ in L-mode and H-mode regimes. Similarities in L-mode discharges between JET and JT-60U are well reproduced by the code as well as the sudden approach to detachment seen in JT-60U when a $1 \%$ chemical sputtering yield for carbon is assumed. Comparisons between similar L-mode regimes in JET, JT-60U and Alcator C-MOD reveal the larger importance of anomalous energy convection in Alcator C-MOD with regard to larger tokamaks. H-mode discharges of DIII-D and JET can be satisfactorily modelled with similar values of the transport coefficients (approximately an order magnitude smaller than in L-mode) when the power flow into the scrape-off layer (SOL) is dominated by the ion channel, as previous EDGE2D simulations for JET suggested. (c) 1999 Elsevier Science B.V. All rights reserved.
\end{abstract}

Keywords: Two-dimensional modelling; B2-EIRENE; Edge modelling; ALCATOR C-MOD; DIII-D; JET; JT-60U

\section{Introduction}

The problems of heat load and erosion on first wall materials have been identified as one of the main areas to be addressed in the design of today's divertor experiments and next step devices such as ITER [1]. Presently, the favoured solutions to these problems are based on the extrapolation of the radiative divertor regimes observed in experiments, where volumetric losses reduce the power and ion flux to the target achieving the detached divertor regime.

\footnotetext{
${ }^{*}$ Corresponding author. Tel.: +49-89 3299 4219; fax: +49-89 3299 4312; e-mail: loartea@sat.ipp-garching.mpg.de
}

Two-dimensional plasma fluid codes coupled to Monte-Carlo or diffusive codes for neutral species such as B2-EIRENE [2,3], EDGE2D-NIMBUS [4,5] and UEDGE $[6,7]$ have been developed to perform realistic calculations of the divertor and scrape-off layer (SOL) plasma parameters. These codes are routinely used to optimise the design of divertors for existing and next step devices. The reliability of these predictions depends on the accuracy of the models used to describe the physical processes that take place in the SOL and divertor region. Therefore, it is crucial to assess the validity of such models to describe the experimental measurements.

In this paper we present the first results of an assessment of the B2-EIRENE code, which is being used extensively to predict the performance of the ITER 
divertor [8-10]. This code has been thoroughly compared with measurements from ASDEX-Upgrade discharges [11-13] and with other experiments [14,15]. In order to carry out this comparison in a more complete fashion, an experimental database has been established (the ITER edge profile database) which contains Lmode and $\mathrm{H}$-mode discharges with attached and detached plasmas from the main divertor experiments: Alcator C-MOD, ASDEX-Upgrade, DIII-D, JET and JT-60U. The creation of this database has been supervised by the ITER Divertor Modelling and Database Expert Group within the framework of the ITER-EDA activities. The complete validation of the B2-EIRENE code against the discharges in the database is a long term process, and at present, far from complete. Also, the detailed characteristics of many experiments can only be studied by the modelling groups at the experiments themselves. The purpose of this study is not to repeat that work, but to achieve simulations that are close enough to the experiment so that the conclusions drawn from them are reliable. Despite this limitation, this study has already shown similarities and differences between different divertor experiments that shed some light into the anomalous transport processes in the SOL. Due to computing power requirements, we have concentrated mostly on simulations without impurities for all devices. Simulations including impurities have only been performed so far for JT-60U L-mode discharges, while the other experiments are presently being studied. We have not attempted to model the pumping/fuelling arrangements in each experiment but, in general, a recycling coefficient of 1 for all surfaces has been assumed. For simulations with impurities we have considered only carbon, which is produced by physical sputtering plus chemical sputtering with an effective yield of $1 \%$ on all surfaces. As usual, anomalous perpendicular transport for particles and energy is described by effective coefficients $\left(D_{\perp}, \chi_{\perp}^{\mathrm{e}}, \chi_{\perp}^{\mathrm{i}}\right)$ constant in space.

\section{Ohmic and L-mode assessment}

Discharges from Alcator C-MOD, JET and JT-60U have been included in this assessment. The discharges from JET and JT-60U correspond to similar main plasma parameters (2 MA L-mode density scans with $\sim 4 \mathrm{MW}$ of additional heating) and can be modelled with similar input parameters. For the JT-60U simulations we have chosen transport coefficients with values used for JET simulations with EDGE2D [16] $\left(D_{\perp}=0.15 \mathrm{~m}^{2} \mathrm{~s}^{-1}, \chi_{\perp}^{\mathrm{e}}=5.0 \mathrm{~m}^{2} \mathrm{~s}^{-1}, \chi_{\perp}^{\mathrm{i}}=5.0 \mathrm{~m}^{2} \mathrm{~s}^{-1}\right)$. The B2-EIRENE simulations for JET Mk I with such parameters show a somewhat broad density profile because of the simplified neutral recycling assumptions used in this study, instead of the more realistic pumping/ puffing scheme used at JET and accounted for in the
EDGE2D simulations [16]. Both the EDGE2D and B2EIRENE simulations display a typical disagreement between model and experiments namely the lack of correct modelling of the inner/outer divertor asymmetries. While the outer divertor is in the high recycling regime in the experiment and the simulations, the inner divertor is more detached in the experiment than in the code, which tends to produce a more symmetric solution. Part of this asymmetry may be associated with the lack of impurities in these JET simulations. Simulations for JT-60U with impurities for plasmas in a similar regime to these JET experiments are satisfactorily modelled with the same transport coefficients (Fig. 1). For this case the asymmetries in the code are again smaller than in the experiment ( $\mathrm{CII}$ and $\mathrm{H}_{\alpha}$ emission). The Langmuir probe data at the inner and outer divertor are well reproduced by the code. This fact together with the good agreement of the measured and calculated $\mathrm{H}_{\alpha}$ emission at the outer divertor but not at the inner divertor, indicates that the inner divertor experimental electron temperature from the probes is overestimated. The level of impurity radiation for JT-60U is well reproduced by assuming carbon production by physical sputtering plus a $1 \%$ effective chemical yield over the whole density scan. The radiated power fraction and the DoD (degree of detachment [17]) comparison between code and experiment is shown in Fig. 2 for simulations with/without impurities. From this figure it is clear that only simulations with impurities describe the approach to detachment properly at the measured separatrix density and the measured radiated power fraction. The difference between the model and experiment here is due to the absence of core radiation in the calculations, such a core radiation is $10-30 \%$ of the input power in the experiment. Furthermore, it is also seen that the window for stable detached operation was very limited in the previous JT-60U divertor geometry as compared for instance to JET with the Mark I divertor [17], which is well reproduced by the sudden increase of the DoD in the simulations (for larger DoDs convergence is not obtained, i.e. 'Code Marfe' appears). Although the similarities between the attached phases of JET and JT$60 \mathrm{U}$ both in code and experiment may seem trivial, they are actually very important because they show that both experiments are consistent and that the models reflect this consistency. The two major disagreements between code and the JT-60U experimental measurements concern the $Z_{\text {eff }}$ and the ion temperature in the SOL. The $Z_{\text {eff }}$ remains around 2 in the experiment for all the density scan [18], while in the simulations it falls from 2.2 (low density) to 1.2 (high density). This implies that either the modelling of main chamber production of impurities or their transport (need for an inwards pinch [19]) is not adequate in these simulations. Our simulations have been carried out assuming the same power across the separatrix in the ions as in electrons (2 MW 

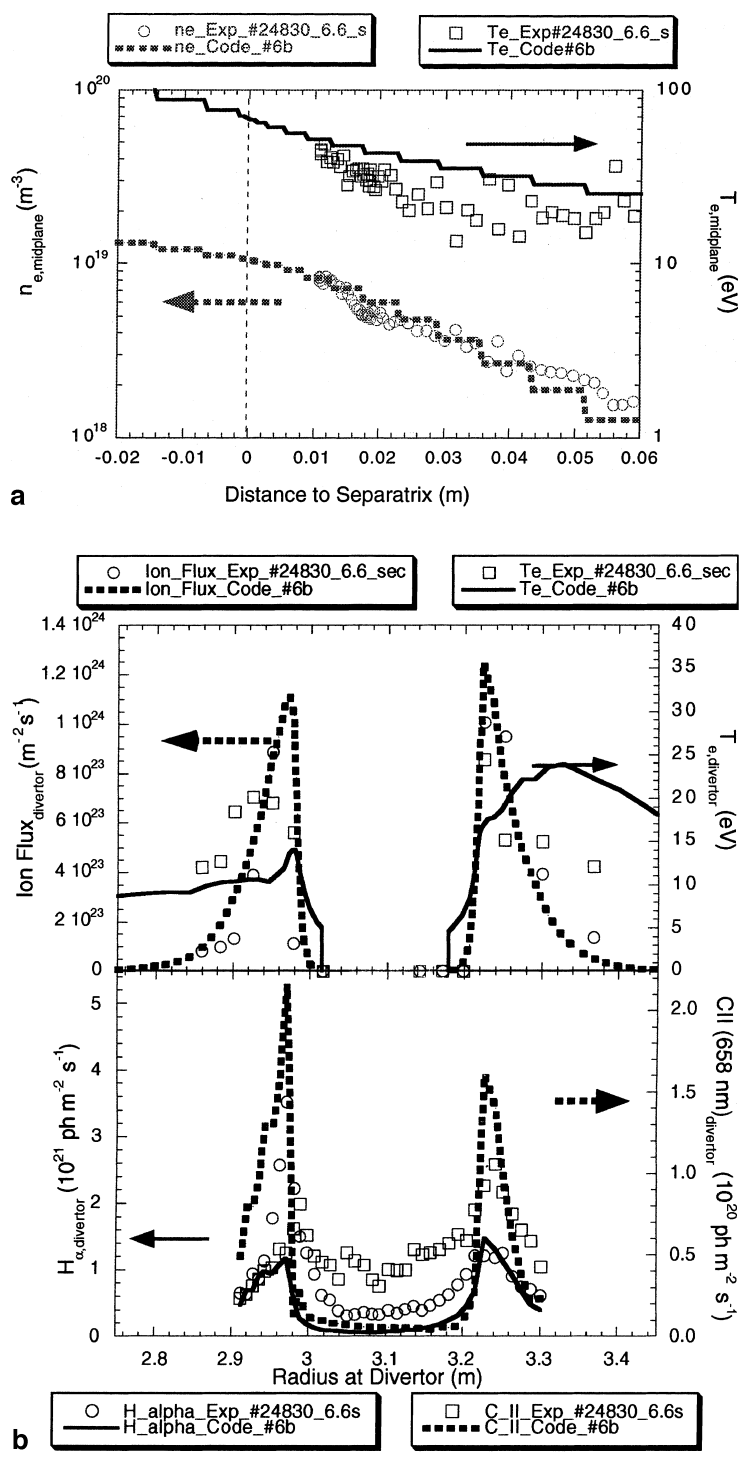

Fig. 1. (a) Midplane density and electron temperature profiles for a JT-60U L-mode discharge and the simulation with B2EIRENE. (b) (Top) Divertor ion flux and electron temperature profiles for a JT-60U L-mode discharge and the simulation with B2-EIRENE. (c) (Bottom) Divertor $\mathrm{H}_{\alpha}$ and CII emission profiles for the same discharge/simulation.

per channel) and the same $\chi_{\perp}$ for both. This choice leads to $T_{\mathrm{i}}^{\mathrm{SOL}} \approx 2 \times T_{\mathrm{e}}^{\mathrm{SOL}}$ in the calculations, while the measured ratio [18] is close to 3.5 . If these ion measurements are representative of the Maxwellian ions in the SOL, this would imply a smaller $\chi_{\perp}^{\mathrm{i}}$ for the ions in the SOL and/or that the ion heat flux is flux limited in the experiment compared to the simulations, where no flux limit for the ion heat flux is imposed ( 0.2 is used for the electrons). A sound theoretical basis for the ion heat flux

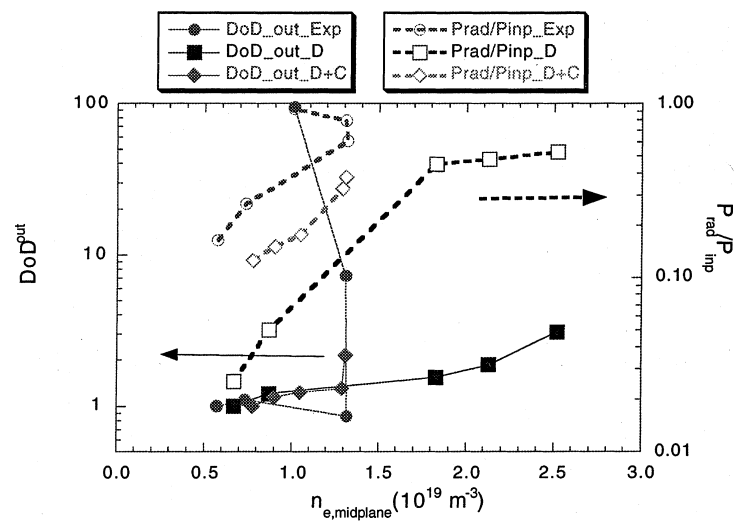

Fig. 2. Degree of detachment for the outer divertor separatrix and radiation fraction for JT-60U and the B2-EIRENE simulations with $(\mathrm{D}+\mathrm{C})$ and without $(\mathrm{D})$ impurities.

limit does not yet exist and should be developed in order to make progress in this area.

Modelling of Alcator C-MOD plasmas in equivalent ohmic regimes shows a fairly different behaviour. Such discharges can only be modelled with the code if remarkably different transport coefficients are used compared to those for JET and JT-60U, namely, $\left(D_{\perp}=0.2 \mathrm{~m}^{2} \mathrm{~s}^{-1}, \chi_{\perp}^{\mathrm{e}}=0.04 \mathrm{~m}^{2} \mathrm{~s}^{-1}, \chi_{\perp}^{\mathrm{i}}=0.04 \mathrm{~m}^{2} \mathrm{~s}^{-1}\right)$ and a low recycling coefficient is used at the divertor. With these prescriptions, the SOL energy transport in CMOD seems to be dominated by convection across the field rather than conduction, as first pointed out in [14]. The existence of a sizeable main chamber particle source has recently been identified in Alcator C-MOD [20]. This source is believed to be caused by direct recycling on the walls and not neutral leakage from the divertor. Therefore, the method to simulate such a source in our calculations leads naturally to an overestimate of the convected power for Alcator C-MOD and to a too low level of main chamber recycling (compared to $\mathrm{H}_{\alpha}$ measurements). An example for such simulation is given in Fig. 3, where the recycling coefficient at the divertor has been reduced to 0.8 . With such a prescription, the effective particle source in the plasma can be compared to the total number of ionisations in the computational domain for JET, JT-60U and Alcator C-MOD where it is much larger (Fig. 4). This automatically leads to the convective anomalous transport to dominate the SOL in C-MOD, while it represents a minor contribution in JET and JT-60U (Fig. 4). It is expected that simulations that include the proper modelling of the by-pass leakage from the divertor and direct main chamber recycling will have a lower convected power. However, it is already clear that the level of convected power in Alcator CMOD will be much larger than that of other experiments [20] as indicated by these simulations. 


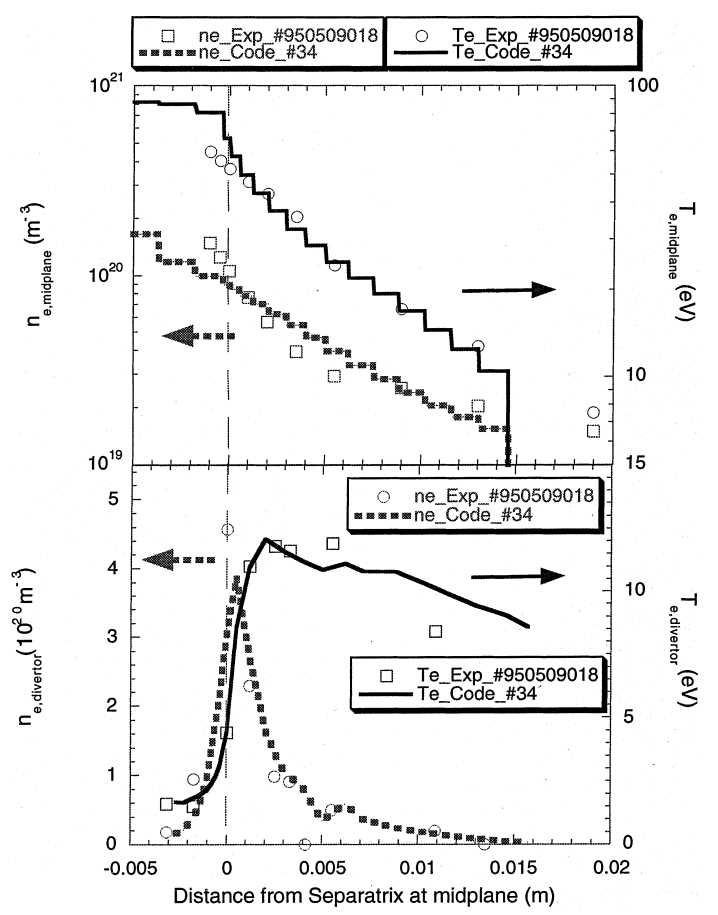

Fig. 3. (Top) Midplane density and electron temperature profiles for an Alcator C-MOD ohmic discharge and the simulation with B2-EIRENE. (Bottom) Divertor electron density and temperature profiles for the same discharge/simulation.

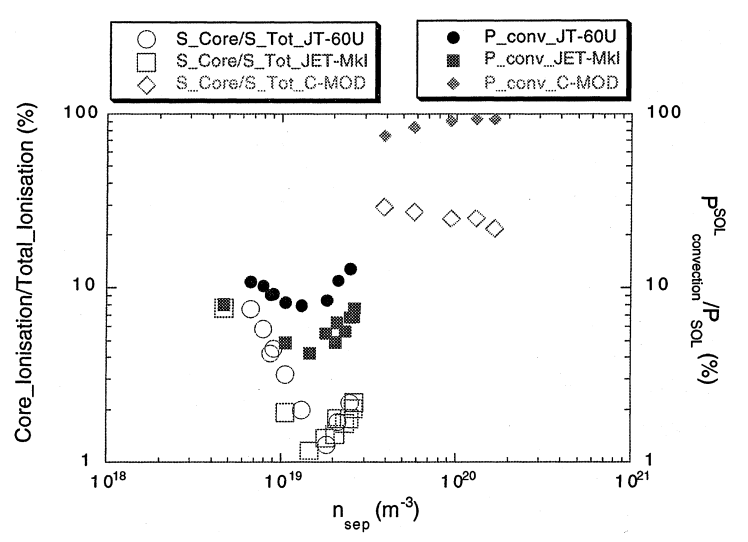

Fig. 4. Core plasma particle source ( $\%$ normalised to total ionisation source) and fraction of convected power into the SOL $(\%)$ for JET, JT-60U and Alcator C-MOD ohmic/L-mode simulations.

Another difference between Alcator C-MOD with respect to JET and JT-60U is resolved as well by the simulations without any special prescription. For Lmode attached discharges in Alcator C-MOD [21], it is found experimentally that the electron pressure at the midplane is approximately a factor of 2 larger than at the divertor, while in JET [22,23] and JT-60U [18] approximate electron pressure conservation is found. This difference is naturally reproduced by the calculations. It is due to momentum conservation in attached plasmas, the sheath boundary conditions and the simulation results: $T_{\mathrm{i}}^{\mathrm{SOL}} \approx(1.1-1.2) \times T_{\mathrm{e}}^{\mathrm{SOL}}, T_{\mathrm{i}}^{\mathrm{DIV}} \approx T_{\mathrm{e}}^{\mathrm{DIV}}$ for $\mathrm{C}-$ MOD and $T_{\mathrm{i}}^{\mathrm{SOL}} \approx 2 \times T_{\mathrm{e}}^{\mathrm{SOL}}, T_{\mathrm{i}}^{\mathrm{DIV}} \approx T_{\mathrm{e}}^{\mathrm{DIV}}$ for $\mathrm{JET} / \mathrm{JT}-$ $60 \mathrm{U}$.

\section{H-mode assessment}

We have concentrated on low density ELMy Hmodes in JET and DIII-D for the plasma state in between ELMs. The results with B2-EIRENE for JET are similar to those obtained with EDGE2D for JET [23,24]. In these studies it was found that, in order to reproduce the experimental parameters at the divertor (ion flux and $T_{\mathrm{e}}$ ) and the SOL power flow, it is necessary not only to use very low diffusion coefficients but to further assume that most of the SOL power flows via the ion channel in low density H-modes [22-24]. Such results have been confirmed in B2-EIRENE simulations of DIII-D discharges, where infrared power measurements are available (see Fig. 5). The split of the power flux into the SOL used in these simulations is $P_{\mathrm{i}}^{\mathrm{SOL}} / P_{\mathrm{e}}^{\mathrm{SOL}} \approx 4$ with transport coefficients $\quad\left(D_{\perp}=0.01 \mathrm{~m}^{2} \mathrm{~s}^{-1}, \chi_{\perp}^{\mathrm{e}}=\right.$ $\left.0.7 \mathrm{~m}^{2} \mathrm{~s}^{-1}, \chi_{\perp}^{\mathrm{i}}=0.35 \mathrm{~m}^{2} \mathrm{~s}^{-1}\right)$. These values are similar to those used for the JET modelling [23,24] and to those from UEDGE modelling for such discharges in DIII-D [25]. It is important to note that the very peaked electron temperature profile at the divertor is well reproduced with the matched upstream electron temperature and density profiles. For these calculations the ion temperature in the SOL is underestimated in the simulations, as found for JT-60U L-modes. In this case a further reduction of $\chi_{\perp}^{\mathrm{i}}$ can be excluded, as it would affect the power deposition profile in the divertor, which is well reproduced with the value above. The conclusion to be drawn is therefore that if $T_{\mathrm{i}}^{\mathrm{SOL}}$ charge-exchange measurements are representative of the Maxwellian ions, then the ion heat flux along the field is strongly flux limited (no ion flux limit has been imposed in these simulations). As already mentioned, a sound theoretical basis for the ion heat flux limit remains to be developed.

\section{Conclusions}

In this paper we have summarised the assessment of the models contained in B2-EIRENE by comparing simulations with discharges from existing experiments. So far, most of the simulations have been performed without impurities, which are known to be important in the approach to detachment. It is, however, clear that JT-60U and JET MkI attached L-mode regimes are 

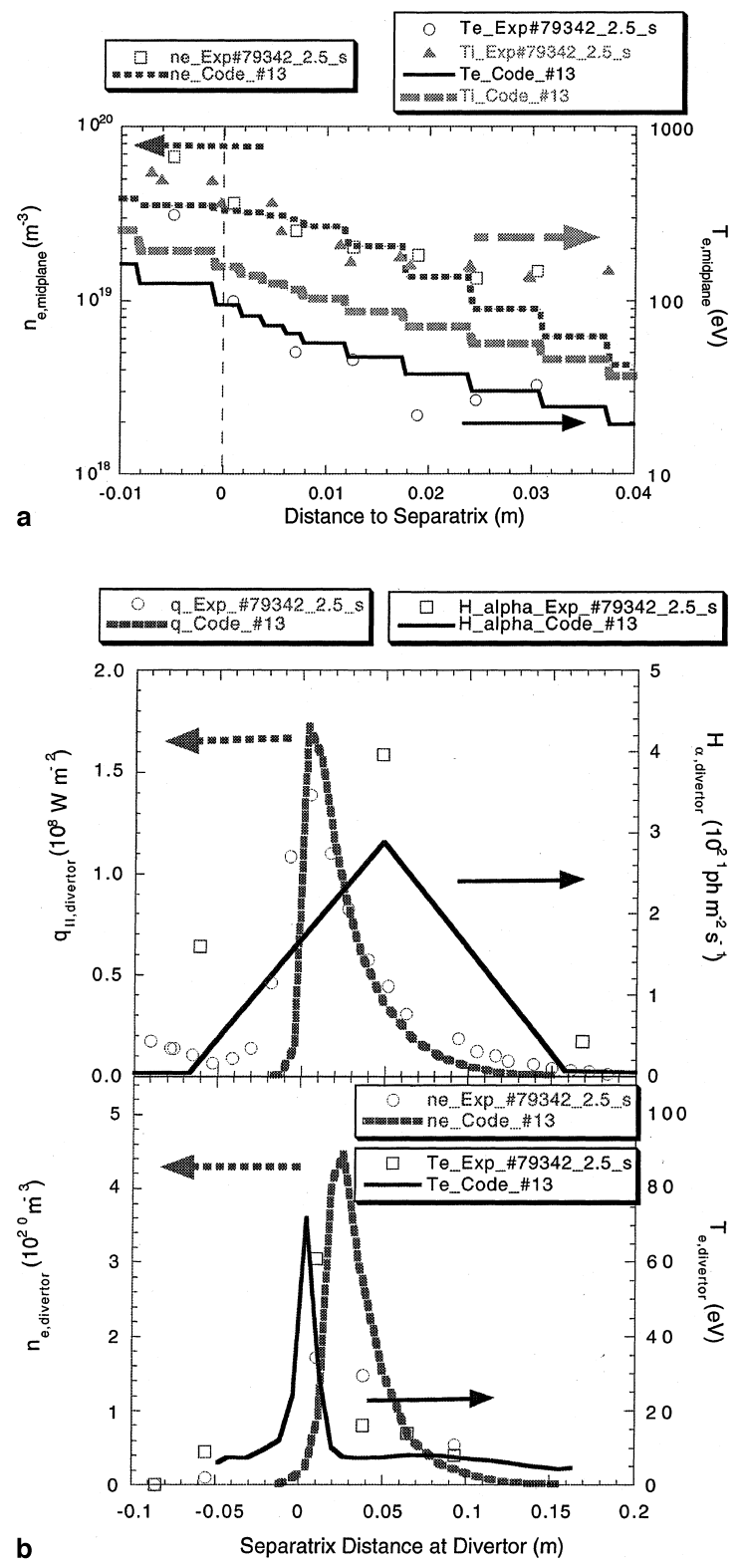

Fig. 5. (a) Midplane density and electron + ion temperature profiles for a DIII-D low density ELMy H-mode discharge and the simulation with B2-EIRENE. (b) (Top) Power flux and $\mathrm{H}_{\alpha}$ profiles at the outer divertor for a DIII-D low density ELMy Hmode discharge and the simulation with B2-EIRENE. (b) (Bottom) Electron density and temperature at the outer divertor for the same discharge/simulation.

consistently modelled by the code. The sudden approach to detachment in the JT-60U open divertor is well reproduced by the code (simulations with impurities). Although no calculations with impurities have been yet performed for JET with B2-EIRENE, it is expected that the more gradual approach to detachment will be re- produced by the code, as it has been the case for ASDEX-Upgrade [13] and for JET with EDGE2D [16]. Modelling of similar discharges for Alcator C-MOD reveals the need for a substantially large main chamber source. This is achieved in the simulations by lowering the recycling at the divertor, which leads to the anomalous transport in the Alcator C-MOD SOL to be convection rather than conduction dominated, as it is the case in JET and JT-60U for similar discharges. Although the low recycling coefficient used in the simulations is not realistic, the larger importance of convection in the Alcator C-MOD SOL is a robust conclusion from this study. Therefore, the difference in the anomalous transport coefficients used in the Alcator C-MOD simulations may not be related to a large size scaling of the heat diffusivity but to an altogether different balance between convection and conduction in the Alcator CMOD SOL, compared to JET and JT-60U.

Simulations of low density ELMy H-modes for JET and DIII-D have shown many similarities. The best fit to the experiment is achieved by assuming much smaller transport coefficients than those used for L-mode and with the power flow into the SOL being dominated by the ion channel, as earlier analysis of the JET data and simulations with EDGE2D for JET indicated. The values of the transport coefficients needed to simulate JET and DIII-D are virtually the same, indicating the lack of size scaling for anomalous transport at the plasma edge in H-modes.

The work presented here is the first report on the assessment of B2-EIRENE modelling by using discharges in the ITER edge profile database. This work will now proceed with studies including carbon impurities for all device/regimes in the database and by implementing more realistic neutral recycling assumptions, each fitted to the characteristics of the specific machine. The results of such a study will establish rules for the scaling of anomalous transport among different size machines which will provide an experimentally based extrapolation of the anomalous transport coefficients to ITER. The divertors modelled in this paper are geometrically open (but Alcator C-MOD). In future, data from more closed divertor experiments, such as the MkII divertor in JET or the W shape divertor in JT$60 \mathrm{U}$, will be added to the database in order to assess the code in these more ITER relevant divertor configurations.

\section{Acknowledgements}

The authors wish to acknowledge fruitful discussions with members of the ITER Divertor Modelling and Database Expert Group and ITER Divertor Physics Expert Group. The database used in the present analysis was supplied by tokamak teams all over the world: 
Alcator C-MOD, DIII-D, JT-60U and JET. The authors wish to express their sincere thanks to all members of these teams. Computational support by the IPP Computing Department and the computer support staff of the ITER Garching JWS is also acknowledged.

\section{References}

[1] R. Parker et al., J. Nucl. Mater. 241-243 (1997) 1.

[2] B.J. Braams, Computational studies in tokamak equilibrium and transport, PhD thesis, University of Utrecht, 1986.

[3] D. Reiter et al., Plasma Phys. and Control. Fusion 33 (1991) 1579.

[4] R. Simonini et al., J. Nucl. Mater. 196-198 (1992) 369.

[5] R. Simonini et al., Contrib. Plasma Phys. 34 (1994) 368.

[6] T.D. Rognlien et al., J. Nucl. Mater. 196-198 (1992) 347.

[7] T.D. Rognlien, Contrib. Plasma Phys. 36 (1996) 105.

[8] A.S. Kukushkin et al., J. Nucl. Mater. 241-243 (1997) 268.

[9] A.S. Kukushkin et al., Contrib. Plasma Phys. 38 (1998) 20.

[10] H.D. Pacher et al., these Proceedings.

[11] H.S. Bosch et al., J. Nucl. Mater. 220-222 (1995) 558.

[12] R. Schneider et al., Proceedings of the 22nd EPS Conference on Controlled Fusion Plasma Physics, vol. 19C, Part 4, 1997, p. 285.
[13] D.P. Coster et al., Proceedings of the 24th EPS Conference on Controlled Fusion Plasma Physics, vol. 21A, Part 4, 1997, p. 1437.

[14] R. Vesey et al., Bull. Am. Phys. Soc. 40 (1995) 1702.

[15] A. Hatayama et al., Proceedings of the 24th EPS Conference on Controlled Fusion Plasma Physics, vol. 21A, Part 2, 1997, p. 505.

[16] A. Loarte, J. Nucl. Mater. 241-243 (1997) 118.

[17] A. Loarte et al., Nucl. Fusion 38 (1998) 331.

[18] N. Asakura et al., J. Nucl. Mater. 241-243 (1997) 559.

[19] S. de Peña Hempel et al., Proceeedings of the 24th EPS Conference on Controlled Fusion Plasma Physics, vol. 21A, Part 4, 1997, p. 1401.

[20] M. Umansky, S. Krasheninnikov, B. LaBombard, J.L. Terry, Comments on particle and energy balance in the edge plasma of Alcator C-Mod, Phys. Plasmas, submitted.

[21] B. LaBombard et al., J. Nucl. Mater. 241-243 (1997) 149.

[22] A. Loarte, Estudio de los flujos de energía y partículas en el borde del plasma del tokamak JET, PhD thesis, Universidad Complutense de Madrid, 1992.

[23] A. Loarte et al., J. Nucl. Mater. 220-222 (1995) 606.

[24] G.K. McCormick et al., J. Nucl. Mater. 241-243 (1997) 444.

[25] G.D. Porter et al., Contrib. Plasma Phys. 34 (1994) 454. 